\title{
Frequency-Dependent Focal Adhesion Instability and Cell Reorientation Under Cyclic Substrate Stretching
}

\author{
Yuan Zhong, ${ }^{1}$ Dong Kong, ${ }^{2}$ Lanhong Dai, ${ }^{2}$ and BaOhua $\mathrm{JI}^{1}$ \\ ${ }^{1}$ Biomechanics and Biomaterials Laboratory, Department of Applied Mechanics, Beijing Institute of Technology, \\ Beijing 100081, China; and ${ }^{2}$ State Key Laboratory of Nonlinear Mechanics, Institute of Mechanics, Chinese Academy \\ of Sciences, Beijing 100190, China
}

(Received 22 December 2010; accepted 8 July 2011; published online 28 July 2011)

Associate Editors Mian Long and Fan Yuan oversaw the review of this article.

\begin{abstract}
The adhesion-based cell mechanosensitivity plays central roles in many physiological and pathological processes. Recently, quantitative understanding of cell responses to external force has been intensively pursued. However, the frequency dependent cell responses to the substrate stretching have not yet been fully understood. Here we developed a multiscale modeling framework for studying cell reorientation behaviors under substrate stretching, in which the mechano-chemical coupling at molecular, subcellular, and cellular scales was considered. The effect of matrix stiffness was also considered in a FEM based mechano-chemical coupling simulation. We showed that the collapsing time of focal adhesion decreases with the increasing of the loading frequency, however, the cell reorientation time exhibits a biphasic frequency-dependent behavior. Our results suggested that this biphasic behavior might be caused by the competition between the frequency-dependent collapsing of focal adhesions and the less frequency-dependent formation of stress fibers aligning away from the loading direction. At the low loading frequency, the collapsing of focal adhesion dominates the reorientation process, however, at the high loading frequency the polymerization of stress fiber dominates the reorientation. Moreover, we showed that the compliance of matrix may help accelerate the cell reorientation because focal adhesion is prone to be instable on soft matrix.
\end{abstract}

Keywords-Cell adhesion, Mechanosensitivity, Stress fiber, Mechano-chemical coupling, Multiscale modeling, Loading frequency.

\section{INTRODUCTION}

Adhesion-based cell mechanosensitivity plays a central role in not only physiological processes, ${ }^{10,25}$ such as

Address correspondence to Baohua Ji, Biomechanics and Biomaterials Laboratory, Department of Applied Mechanics, Beijing Institute of Technology, Beijing 100081, China. Electronic mail: bhji@bit.edu.cn cell differentiation, growth, morphogenesis and migration, but also pathological processes ${ }^{29}$ especially the formation and development of many vital diseases, such as cancer, atherosclerosis and asthma, to name only a few. Cells sense their environments and external stimuli through focal adhesion (FA), and then regulate their behaviors accordingly. In the past two decades, considerable efforts have been paid for understanding the mechanisms of how cells sense these mechanical signals, transfer and transduce them, and then make responses. One typical problem is cells' active reorientation under cyclic substrate stretching. ${ }^{14,16,19,31,34,44,48,56}$ It was found that the behaviors of cell reorientation depend on many parameters of the loading, such as loading frequency, loading mode and loading amplitude. ${ }^{16,48}$ Cell reorientation involves complex internal and external dynamic processes of cells at different spatial and temporal scales, including dynamics of FAs and cytoskeleton remodeling, as well as the cell-matrix interaction. Despite the biological complexity underlying the different cell responses, it was shown that cells follow the basic mechanical principles, e.g., their final (equilibrium) aligning angle can be predicted based on the principle of minimum strain energy, ${ }^{57}$ which suggests that the cell reorientation may be a mechanism for cells to maintain an optimal tension state in cell cytoskeleton. ${ }^{17,34}$ In addition, the threshold value of external loading for cell reorientation can be predicted by assuming a limiting anchoring strength of the FAs. $^{12,36,37}$

Cell reorientation is cell's responses to the external load via the interplay of dynamic responses of FAs and those of actin cytoskeleton. The mechanical properties (elastic and viscoelastic) of cells are mainly determined by the stability of FAs, the mechanical properties of cytoskeleton, and the loading modes of the stimuli. Because there are thousands of different proteins and many protein-protein interactions, there exists a wide 
distribution of characteristic times in cell cytoskeleton. ${ }^{13}$ In addition, actin cytoskeleton (e.g., stress fiber) and focal adhesions are interdependent, ${ }^{22}$ i.e., focal adhesion proteins mediate the key signal transduction events that regulate actin remodeling and contraction, ${ }^{23,53}$ and the internal contractility generated by actin cytoskeleton help the maturation of focal adhesions from nascent focal complexes. ${ }^{52}$ Therefore, cell reorientation requires the coordination of actin cytoskeleton and focal adhesions. More importantly, the force applied at the extracellular matrix (ECM) is transmitted to the actin cytoskeleton through focal adhesions, thus focal adhesions play central roles in regulating actin cytoskeleton remodeling through not only chemical pathways but also mechanical anchors at the cell-matrix interface. As a result, stable focal adhesions are favored by the stability of stress fibers, but unstable focal adhesions will initiate depolymerization of the associated stress fibers. It was shown that the breaking force of stress fiber is on average of $377 \mathrm{nN},{ }^{18}$ which is much higher than that of a focal adhesion on the order of tens of nano-Newton because the breaking force of a single integrin-ligand bond is only on the order of tens of pico-Newton. ${ }^{35}$ Therefore, focal adhesion's rupture might be the trigger of cell reorientation, which firstly causes the depolymerization of the associated stress fibers in the loading direction, and then the polymerization of new stress fibers in other favored directions.

Experimental studies showed that the cell reorientation was frequency dependent. It has been known that cells exhibit different behaviors under dynamic stretching from those under static or quasi-static stretching. ${ }^{17,34}$ Under the static loading, the cells prefer to align along the loading direction instead of making reorientation. Recently, however, much attention has been paid on the effects of loading frequency on the cell reorientation. Liu et $a l .{ }^{44}$ did substrate stretching experiments at different loading frequencies and analyzed the cell responses from several aspects, including the rate of cell reorientation, the $\mathrm{F} / \mathrm{G}$ actin ratio, and activation of integrin, etc. Furthermore, Jungbauer et $a l .{ }^{31}$ showed that not only the cell reorientation was frequency dependent, but also this frequency dependence showed a biphasic way for subconfluent cell system. They showed that at low loading frequency, the cell reorientation time decreases exponentially with the frequency; however when the frequency was higher than a critical value, the reorientation time vs. the frequency curve will approach a plateau, not depending on the frequency any more. To study the mechanisms of the frequency dependence of cell reorientation, Safran and coworkers ${ }^{17}$ developed a force-dipole model in which they can consider the intrinsic relaxation times at subcellular and cellular levels with a phenomenological approach. Their model captured the exponential relationship of the loading frequency vs. cell reorientation time. Inspired by the force dipole model, Kong et al. ${ }^{30,36}$ developed a mesoscopic model that not only considered the dynamics of adhesion molecules, but also accounted for the mechanical properties of stress fiber. They showed that the loading frequency affected FA's stability that consequently affected the cell reorientation. However, these studies did not consider the dynamic turnover of stress fibers during cell reorientation in their models.

Chien and coworkers ${ }^{34}$ showed that the cell reorientation was actually accomplished by stress fiber reorientation through the disassembly of stress fibers along the stretching direction and then the reassembly of those along the direction perpendicular to the stretching. They found that the stretch-induced stress fiber reorientation is a function of the interplay between Rho pathway activity and the magnitude of the stretching loading. Then, Lee et $\mathrm{al}^{43}$ further demonstrated in more details that the stretch-induced SFs reorganization is a complex process involving multiple phenomena including SF disassembly, reassembly, and fusion that depend on the rate of the stretching, Rhokinase and MLCK. Later, to understand the mechanisms of the rapid disassembly of SFs, Matsui et al. ${ }^{47}$ experimentally investigated the effects of MgATP concentration on SFs' shortening and stability.

Kaunas and coworker ${ }^{28,32}$ developed a kinetic model to describe the dynamics of the stress fiber and the stability of FAs. The cell reorientation was modeled by the collective behaviors of remodeling of stress fibers in the cell. Their simulation showed that the remodeling of stress fibers can effectively describe the cell reorientation, and the cyclic loading frequency did affect the rate of the remodeling process. They showed that the rate of stress fiber disassembly determines the rate of reorientation. In addition, they showed that there is a threshold frequency, below which SFs were able to self-adjust in order to maintain SF stretch at a set-point value. As the frequency increases, the cells become increasingly less capable of adjusting to matrix stretch-induced changes. To compensate, SFs become more likely to reorient toward the direction of least perturbation in stretch. Recently, Kaunas et al. ${ }^{33}$ developed a more advanced kinematic model coupling stress fiber dynamics with c-jun $\mathrm{N}$-terminal kinase (JNK) activation in response to matrix stretching. They predicted that different patterns of matrix stretch result in distinct temporal patterns in JNK activation that compared well with experimental results. In the case of cyclic uniaxial stretching, stretch-induced JNK activation slowly subsides as stress fibers gradually reorient perpendicular to the stretch direction. In contrast, JNK activation is chronically elevated in response to cyclic equi-biaxial stretch. 
Previous studies were mostly focused on one specific scale of cell, e.g., either cellular scale or subcellular scale or molecular scale, less efforts were made to the study that connects all scales together, i.e., from the molecular to the subcellular and the cellular scales. Most importantly, the biphasic relationship of cellreorientation time vs. the loading frequency was not studied. The frequency dependent cell behaviors have yet to be fully understood. In addition, most of these models cannot consider the cell-matrix interaction explicitly in their calculations. This work is aimed to explain the microscopic mechanisms of the frequency dependence of cell reorientation and why it exhibits a biphasic character. Our view of this cell behavior is that it might be the result of interplay of dynamics of cells at different spatial and temporal scales. Therefore, a multiscale approach was developed accordingly. The stability of a single FA was first studied by considering the bond forming and breaking of integrin-ligand interaction at molecular level. The handshake between the subcellular level and cellular level was made through the association of dynamics of stress fiber with the stability of FAs. The collective dynamics of stress fibers was used to represent the cell reorientation. The mechanical interaction between the cell and matrix was fully considered by a finite element (FEM) simulation coupling with the dynamics of FAs and stress fibers. Our results showed that the biphasic behavior was caused by the interplay between a frequency dependent instability of FAs which then caused the depolymerization of the associated stress fibers and a frequency independent polymerization of stress fibers aligning away from the loading direction.

\section{METHODS}

\section{A Microscopic Bond-Cluster Model of FA}

In modeling of stability of adherent cell at molecular and sub-cellular level, the FA was considered as a bond cluster consisting of hundreds of integrin-ligand bonds between cell and matrix, as shown in Figs. 1a and $1 \mathrm{~b}$. The bonds were assumed to be normal to the surface at the beginning, and uniformly distributed with spacing $a$ ( $20 \mathrm{~nm}$ in this study). The upper ends of integrins were anchored to the adhesion plaque, and the bottom ends formed bonds with ligands on the matrix. Once the matrix is stretched, shear deformation at cell-matrix interface will be developed, which can induce tension in the bonds and then change their bond state (ruptured or closed). Each closed bond was modeled as a linear elastic spring, $f=k_{\mathrm{b}} \Delta l_{\mathrm{b}}$, where $k_{\mathrm{b}}$ was the bond stiffness, and $\Delta l_{\mathrm{b}}$ was the bond extension. For a closed bond, the bond force $f$ can lower the energy barrier for bond rupture, and then shorten bond's lifetime. A chemical reaction is commonly used (a)

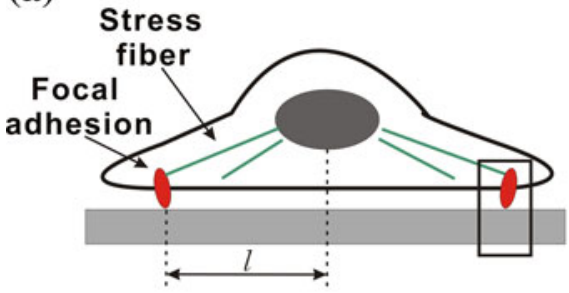

(b)

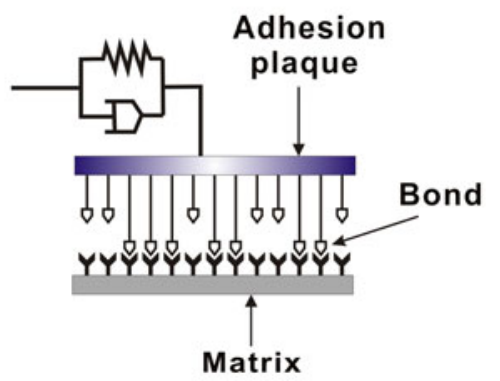

(c)

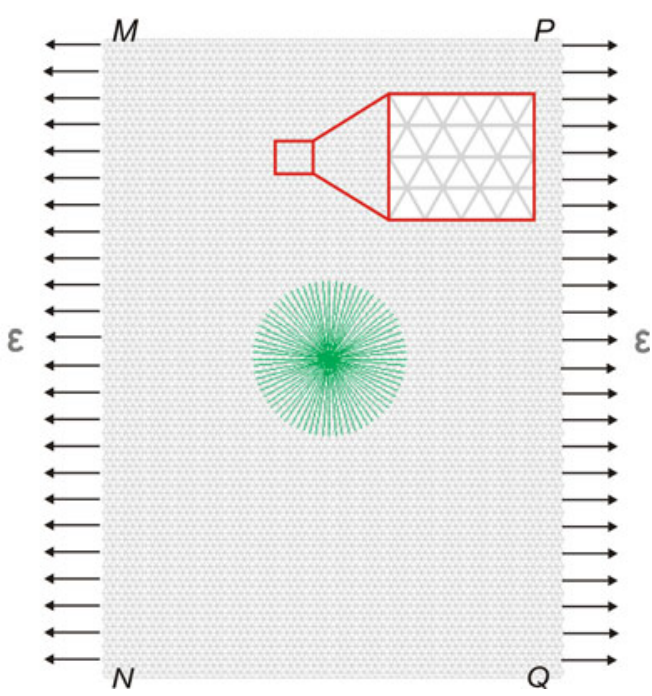

FIGURE 1. A multiscale modeling of cell-matrix system. (a) Illustration of a cell adhering on an elastic matrix via focal adhesions. (b) A bond cluster model of focal adhesion considering rupture and reforming of integrin-ligand bonds and the viscoelasticity of stress fiber. (c) A FEM-based mechano-chemical coupling model of cell reorientation under cyclic substrate stretching acted on the two sides of substrate $M N$ and $P Q$. The cyclic strain was denoted as $\varepsilon$. The substrate was discretized with rod elements in a triangular lattice manner, while the cytoskeleton represented by stress fibers was discretized with cable-like spring elements. The inset in panel $\mathrm{C}$ shows the triangular lattice in a magnified zone. Main parameters in the model were given in Table 1. 
TABLE 1. Main parameters and their values chosen in the calculations.

\begin{tabular}{lllll}
\hline Parameters & Symbol & \multicolumn{1}{c}{ Physiological range } & Used value & References \\
\hline Bond stiffness & $k_{\mathrm{b}}$ & $10^{-2} \sim 10^{1} \mathrm{nN} / \mu \mathrm{m}$ & $10 \mathrm{nN} / \mu \mathrm{m}$ & 5 \\
Bond spacing & $a$ & & $20 \mathrm{~nm}$ & $1,11,45$ \\
Compliance length & $\lambda$ & $0.01 \sim 1 \mathrm{~nm}$ & $0.05 \mathrm{~nm}$ & 20,39 \\
Forward rate constant & $k_{\mathrm{on}}^{0}$ & $1 \sim 100 \mathrm{~s}^{-1}$ & $100 \mathrm{~s}^{-1}$ & 42,51 \\
Reverse rate constant & $k_{\mathrm{off}}^{0}$ & $1 \sim 10 \mathrm{~s}^{-1}$ & $0.01 \mathrm{~s}$ & 4,51 \\
Bond association time & $\tau_{\mathrm{b}}^{-1}$ & $10^{-2} \sim 1 \mathrm{~s}$ & $1.45 \mu \mathrm{N} / \mu \mathrm{m}^{2}$ & 42,51 \\
Modulus of stress fiber & $E_{\mathrm{s}}$ & $0.3 \mu \mathrm{N} / \mu \mathrm{m}^{2} \sim 104 \mu \mathrm{N} / \mu \mathrm{m}^{2}$ & 18 \\
Cross section area of stress fiber & $A_{\mathrm{s}}$ & $3.14 \times 10^{-2} \mu \mathrm{m}^{2} \sim 2.0 \times 10^{-1} \mu \mathrm{m}^{2}$ & $3.14 \times 10^{-2} \mu \mathrm{m}^{2}$ & $18,40,54$ \\
Relaxation time of stress fiber & $\tau$ & $1 \mathrm{~s} \sim 12 \mathrm{~s}$ & $1 \mathrm{~s}$ & 40 \\
\hline
\end{tabular}

for describing the dynamics of adhesion bonds, with reverse rate $k_{\text {off }}$ and forward rate $k_{\text {on }}$. The reverse rate $k_{\text {off }}$ is given by $\mathrm{Bell}^{4}$ as,

$$
k_{\text {off }}=k_{\text {off }}^{0} \exp \left(f \lambda / k_{\mathrm{B}} T\right),
$$

where $k_{\text {off }}^{0}$ is the reverse rate constant in the absence of force, $\lambda$ is viewed as the width of the energy barrier and $k_{\mathrm{B}} T$ is the thermal energy. However, because the Bell's equation is a single energy barrier model, it may ignore other effects that could influence the reverse rate. For example, the catch bond $^{46}$ in fibronectin-integrin complex $^{35}$ can enhance the adhesions. However, we expected that the force for inducing the rupture of focal adhesions should be much larger than the force that triggering the catch bond, and we focused our attention on the effects of the force of larger scale by adopting the simple view of the Bell model. To consider the bond formation, the forward rate $k_{\text {on }}$ can be described as, ${ }^{21,36}$

$$
k_{\mathrm{on}}=k_{\mathrm{on}}^{0} g\left(\tau_{\mathrm{c}}, \tau_{\mathrm{b}}\right) \text { and } g\left(\tau_{\mathrm{c}}, \tau_{\mathrm{b}}\right)=\left\{\begin{array}{ll}
1, & \tau_{\mathrm{c}}>\tau_{\mathrm{b}} \\
\tau_{\mathrm{c}} / \tau_{\mathrm{b}}, & \tau_{\mathrm{c}}<\tau_{\mathrm{b}}
\end{array},\right.
$$

where $k_{\mathrm{on}}^{0}$ is the forward rate constant for an immobile contact, $\tau_{\mathrm{c}}$ is the contact time and $\tau_{\mathrm{b}}$ is the association time of integrin-ligand bonds. The contact time $\tau_{\mathrm{c}}$ is defined as the time during which the free end of the bond is exposed to a contact area that moves with respect to the bond, given by $\tau_{\mathrm{c}}=a /\left(\dot{s}-\Delta \dot{l}_{\mathrm{s}}\right)$, where $a$ is a characteristic length of the contact area (i.e., the spacing between adhesion bonds in the focal adhesion) on the order of tens of nanometers, and $\dot{s}$ and $\Delta \dot{l}_{\mathrm{s}}$ denote the derivatives of the matrix displacement, $s$, and the extension of the stress fiber, $\Delta l_{\mathrm{s}}$, respectively, with respect to time. The definition of $s$ and $\Delta l_{\mathrm{s}}$ will be given later in the text. The intrinsic association time $\tau_{\mathrm{b}}$ is normally the reciprocal of the forward rate constant, and varies inside the interval of $0.01 \sim 1 \mathrm{~s}^{42,51}$ According to Eq. (2), the change of $\tau_{\mathrm{b}}$ can affect the average value of $g\left(\tau_{\mathrm{c}}, \tau_{\mathrm{b}}\right)$, but will not affect the range of its value which is from 0 to 1 . Without losing generality, we chose $\tau_{\mathrm{b}}=0.01 \mathrm{~s}$ in our calculations.
The state of individual bonds is denoted by the state parameter $q$, where $q=1$ corresponds to a closed bond, while $q=0$ corresponds to a ruptured bond.

It was noteworthy that the experimental and theoretical studies showed that the applied force can induce the clustering of integrin-ligand bonds, which then induce growth of FA and consequently enhance its stability. Recently, Kong et al. ${ }^{38}$ proved that the force for inducing the rupture of FA is much larger than that for inducing FA's growth. Here we focused on the frequency dependent instability of mature FA under substrate stretching without explicitly considering clustering of integrin-ligand bonds for simplicity.

To consider the effect of cytoskeleton, a stress fiber was included in this microscopic model. One end of the stress fiber is attached to the bond cluster via adhesion plaque, and the other end is connected with the nucleus of cell. ${ }^{2,52}$ A conceptual Kelvin-Voigt model was adopted to describe the viscoelastic behaviors of the stress fiber, ${ }^{40}$

$$
F=k_{\mathrm{s}} \Delta l_{\mathrm{s}}+\mu \frac{\partial \Delta l_{\mathrm{s}}}{\partial t},
$$

where $F$ is the tension force, $\Delta l_{\mathrm{s}}$ is the extension, $k_{\mathrm{s}}$ and $\mu$ are elastic and damping coefficients of the stress fiber, respectively. There exists an intrinsic relaxation time of the stress fiber, $\tau_{\mathrm{s}}=\mu / k_{\mathrm{s}}$, which is on the order of seconds. ${ }^{40}$

The displacement of matrix at position where the FA anchors has a simple relationship with the strain applied on the matrix as $s=l \varepsilon$, where $l$ is the distance between the focal adhesion and the nucleus of cell along loading direction, as shown in Fig. 1a. The dynamic strain $\varepsilon$ is given by $\varepsilon=\varepsilon_{0}|\sin (\pi \omega t)|$, where $\varepsilon_{0}$ is the strain amplitude, and $\omega$ is the loading frequency. Considering the equilibrium of the adhesion plaque, i.e., the equilibrium of the forces acted by the stressed bonds with that by the stress fiber, we have

$$
F=\sum q_{i} f_{i},
$$

where the subscript ' $i$ ' denotes the $i$-th bond. Equation (4) gives the relationship between the force of stress 
fiber and the forces acting on the bonds of the associated FA.

A governing equation of the bond rupture and formation was introduced for describing the state evolution of bonds in terms of the bond state index $q$. According to Filippov et al., ${ }^{21}$ this equation was written as,

$$
\begin{aligned}
q(t+\Delta t)= & q(t)-q(t) H\left(\xi-k_{\mathrm{off}} \Delta t\right) \\
& +(1-q(t)) H\left(\xi-k_{\mathrm{on}} \Delta t\right),
\end{aligned}
$$

where $\xi$ is a random variable generated randomly in $(0,1)$ and $H$ is a Heaviside step function. For example, in the time period $t \sim t+\Delta t$, a ruptured bond will close if $0 \leq \xi \leq k_{\mathrm{on}} \Delta t$ and a closed bond will rupture if $0 \leq \xi \leq k_{\mathrm{off}} \Delta t$. The bond states of all bonds were tracked in the stochastic simulation (Eq. (5)). The numerical scheme can be found in previous study. ${ }^{36}$ Due to the stochastic characteristics of the simulations, the product results were obtained from an average of 200 independent simulations.

In each FA modeled by the bond cluster, we have 500 bonds connecting the cell to the extracellular matrix. This number of bonds was estimated by considering the size of FA being about 2 micrometer size and the bond spacing being several tens of nanometers.

\section{Mechano-Chemical Coupling Model of Cell-Matrix System}

To simulate the reorientation of cell, a mechanochemical coupling model of cell-matrix system was developed to consider not only the stability of focal adhesion but also the dynamics of turnover of cytoskeleton (stress fiber). In this study, both the cell and matrix were considered as deformable elastomer, i.e., the contractile force by stress fiber can deform the matrix and the substrate stretching can cause the deformation of the cell as well.

\section{Matrix}

We modeled the membrane-like matrix as a twodimensional lattice network of elastic rods in a regular triangular lattice, ${ }^{9}$ as shown in Fig. 1c. The joining points of the rods are called nodes. There are six rods going out of a node in the lattice. In the rod model, the force acting on a node due to the deformation of a rod $\operatorname{reads}^{26,49}$

$$
F_{\mathrm{m}}=E_{\mathrm{m}} A_{\mathrm{m}}\left(\frac{L_{\mathrm{m}}}{L_{\mathrm{mr}}}-1\right)
$$

where $E_{\mathrm{m}}$ is the Young's modulus of the rod, $A_{\mathrm{m}}$ is the cross section area, and $L_{\mathrm{m}}$ is the length of the rod, and $L_{\mathrm{mr}}$ is the rest length of the rod.

\section{Stress Fiber}

It was shown that the mechanical properties of cell are mainly determined by the cytoskeleton. Here we further assumed that the reorientation of cell was mainly accomplished by remodeling of the adhesion related stress fibers, one main type of cytoskeleton. This assumption is consistent with previous experiments and numerical studies, where the stress fibers in the cells were modeled as the load-bearing fibers constrained to deform together as the cell deforms. ${ }^{28,32}$ Because well-spread cells are generally very flat in areas other than the nucleus and the large stress fibers in non-muscle cells are typically localized at the ventral surface, ${ }^{27}$ the stress fibers were assumed to be confined to a two-dimensional plane immediately adjacent to the matrix surface, as shown in Fig. 1c.

Each stress fiber was modeled as a viscoelastic cable as

$$
F_{\mathrm{S}}= \begin{cases}E_{\mathrm{S}} A_{\mathrm{s}}\left(\frac{l_{\mathrm{s}}}{l_{\mathrm{sr}}}-1\right)+u \frac{\partial \Delta l_{\mathrm{s}}}{\partial t} & l_{\mathrm{s}} \geq l_{\mathrm{sr}} \\ 0 & l_{\mathrm{s}}<l_{\mathrm{sr}}\end{cases}
$$

where $E_{\mathrm{s}}$ and $A_{\mathrm{s}}$ are the Young's modulus and the cross section area, respectively, of the stress fiber. $l_{\mathrm{s}}$ and $l_{\mathrm{sr}}$ are the length and the rest length of each stress fiber, respectively. Equation (7) is an alternative expression of Eq. (3) used in the FEM simulation, where $k_{\mathrm{s}}$ in Eq. (3) is equal to $E_{\mathrm{s}} A_{\mathrm{s}} / l_{\mathrm{sr}}$ in Eq. (7). It indicates that the stress fiber can only sustain the tension force, but can not sustain the compression force.

We realized the work by Peterson et al. ${ }^{50}$ showing simultaneous stretching and contraction in stress fiber. However, most of experiments showed that the stress fibers were in general in tension. ${ }^{15,18,40}$ In our study, we simply consider the average behavior of stress fibers, therefore we assumed that the stress fiber is in average in tension.

Because the stress fiber and focal adhesions are interdependent, ${ }^{22}$ the dynamics of turnover of stress fiber is closely related with the stability of FAs. Once substrate is stretched, it will displace the stress fiber through adhesion bonds at FAs, as shown in Figs. 1a and $1 \mathrm{~b}$. Previous study ${ }^{38}$ showed that the responses of FAs to the stretching have the "stabilizing to disruptive" transition as the applied force increases. This observation suggested that there is an optimum region of the force value in which the FAs are stable. However, if the force deviates from this optimum region, the FAs will start to disassemble. The probability of the disassembling of FAs can be described by an empirical relation according to previous studies ${ }^{28,38}$ as

$$
P=k^{i} \Delta t
$$

where $\Delta t$ is the simulation step, and $k^{i}$ is the rate parameter given by 


$$
k^{i}= \begin{cases}k_{0}+k_{1}\left[\left(F_{i}-F_{0}\right) / F_{0}\right]^{2} & F_{i}<F_{0} \\ k_{0}+k_{2}\left[\left(F_{i}-F_{0}\right) / F_{0}\right]^{2} & F_{i} \geq F_{0}\end{cases}
$$

where $F_{i}$ is the force applied on FAs by stress fiber, and $F_{0}=5 \mathrm{nN}$ is the optimum force value for stable FAs at which the shear stress on FAs is about $5 \mathrm{kPa}$, as shown by previous studies, ${ }^{3,6,7,55}$ and $k_{0}=3.0 \times 10^{-4} \mathrm{~s}^{-1}$. Figure 2 shows the probability of disassembly of FAs as the function of force applied on it. We can see that when the deviation of the force from the optimum value $F_{0}$ is larger than a certain level, the FAs will become instable. Parameters $k_{1}$ and $k_{2}$ describe the sensibility of FAs to the deviation of the force from the set-point $F_{0}$. If we assume that the lower limit of the force is $4 \mathrm{nN}$ (i.e., the FA size is $0.8 \mu \mathrm{m}^{2}$ ), and the upper limit is $7 \mathrm{nN}$ (i.e., the FA size is $1.4 \mu \mathrm{m}^{2}$ ), we can obtain the value of $k_{1}$ and $k_{2}$ as $7.5 \times 10^{3} \mathrm{~s}^{-1}$ and $1.875 \times 10^{3} \mathrm{~s}^{-1}$, respectively. The biophysical basis behind Eq. (9) and Fig. 2 was given by previous studies $^{38}$ that there were two force limits which set three regions of force for different states of stability of FAs. When the force is lower than the lower limit, the integrins can not be activated and therefore the FAs are small and unstable; on the other hand, when the force is higher than the upper limit, FAs will disassemble because of bond rupture. Only when the force is at the intermediate value between the two limits, the FAs are stable.

Experiments showed that the value of shear stress on FAs can be somewhat different in different kinds of cells, ${ }^{3,6,7,55}$ e.g., $5.5 \mathrm{kPa}$ on fibroblasts, while $2-5 \mathrm{kPa}$ on cardiac myocytes, and $4.8 \mathrm{kPa}$ on smooth muscle cells, etc. In addition, experiments also showed that the shear stress are different in different FA phases. ${ }^{24}$ Nevertheless, it is broadly accepted that the shear

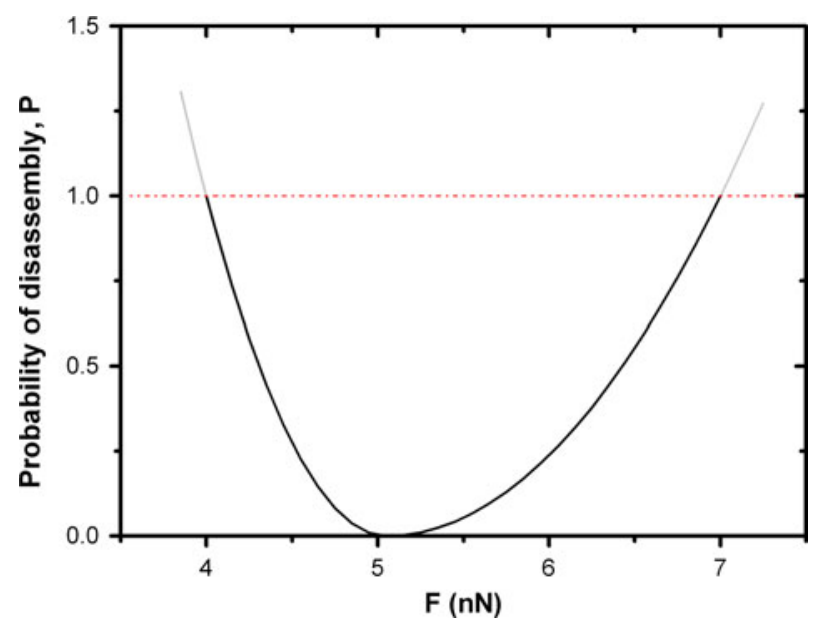

FIGURE 2. The probability of disassembly of FAs at different force values. The responses of focal adhesions can have stabilizing to disruptive transition under the applied force of different magnitude according to previous studies. ${ }^{38}$ stress on FAs is on average about $5 \mathrm{kPa},{ }^{8}$ called the homeostatic stress set-point of adherent cell. Therefore, we adopted the value of $5 \mathrm{kPa}$ in our model.

We note that the force applied on FAs is equal to their size $A$ multiplied by the shear stress on them $\tau$, i.e., $F=\tau A$. In our model, the size of focal adhesions can change in the range of $0.5-10 \mu \mathrm{m}^{2}$, depending on the force applied on them, which is consistent with experiments which showed that the bigger the size of focal adhesions, the higher the force on them, and the ratio between the force and the FA size is nearly constant. $^{7,24}$

When the stress fiber is anchored to the matrix via FAs, its contractile force will deform the matrix. Vice versa, the deformation of matrix will change the structure of actin-cytoskeleton. This mutual interaction between cell and matrix at their interface were solved with the FEM simulation coupled with computation of turnover of FAs and stress fibers. For simulation of cell reorientation, the stress fibers of which associated FAs are stable will perform polymerization. The polymerization rate of those stress fibers was assumed as constant.

On the other side, when FAs become unstable, the stress fiber will perform depolymerization. The depolymerization rate is given by

$$
v_{-}=v_{-}^{\max }\left(1-e^{-\left|F-F_{0}\right| / F^{*}}\right)
$$

where $v_{-}^{\max }$ is the saturation value of the depolymerization rate, and $F^{*}$ is a characteristic force value of stress fiber's depolymerization.

We noted that the FAs of living cell can still remodel, grow and shrink in the absence of external stretching. These kinds of dynamics could be caused by the changing of either the internal contractile force of cells or the internal and external chemical signals. However, in the stretching experiments we modeled in this study, the force applied on the cell could be much larger than the contractile force or the effect of chemical signals so that the stretching force could even cause the disassembly of FAs and then the cell reorientation (see previous works ${ }^{36,38}$ ), particularly at high loading frequency. Therefore, in this work, we assumed that the effect of these changing of the contractile force and chemical signals were so small compared with the external stretching force that they could be neglected.

\section{Simulation Procedure}

The simulation procedure is illustrated in Fig. 3. Firstly, a pre-strain was applied to the stress fibers. Here the applied pre-strain is equal to 0.1 according to the experiments which showed that the active 


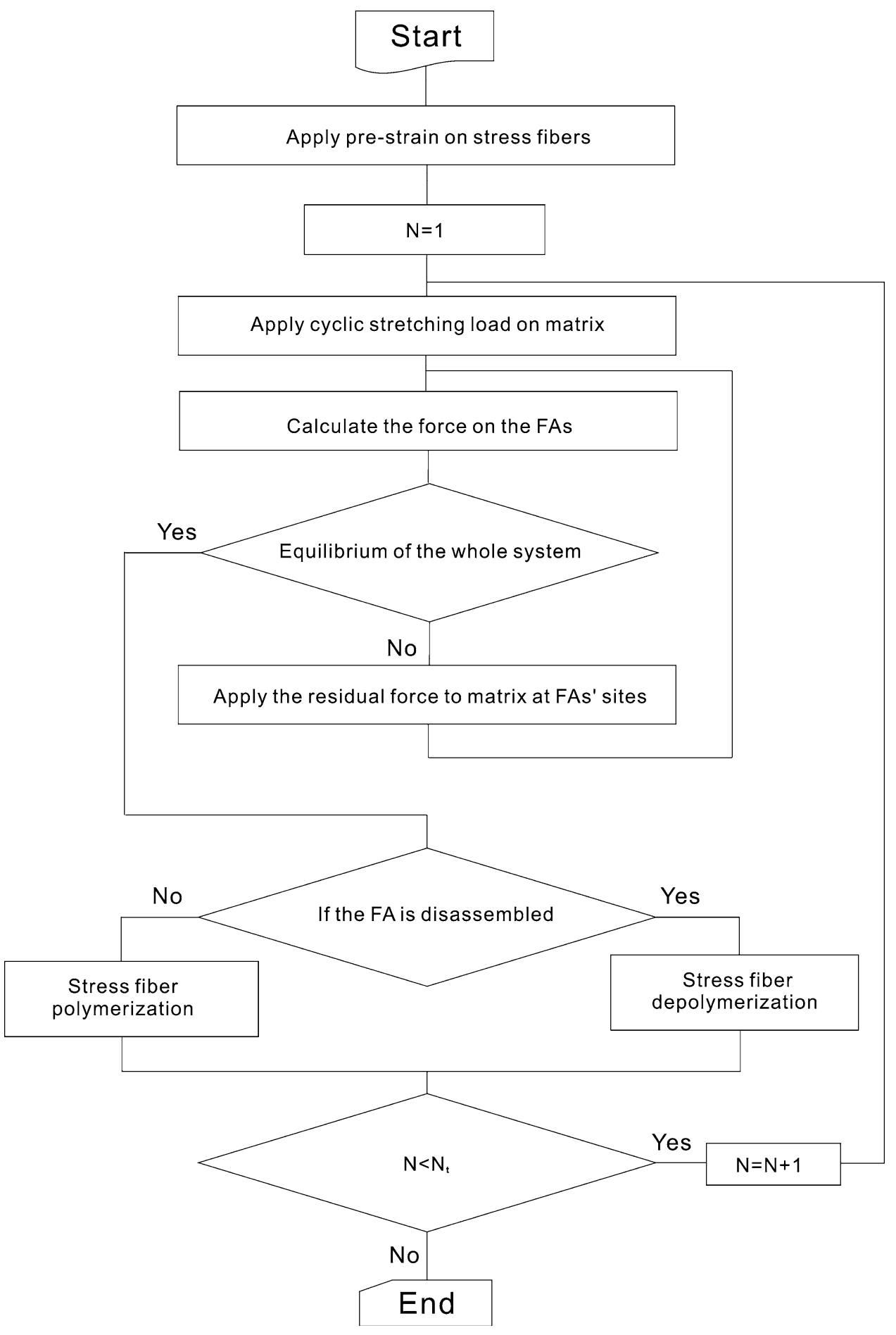

FIGURE 3. Illustration of procedure of the FEM-based mechano-chemical coupling simulations considering both cell-matrix interaction and dynamics of FAs and stress fibers.

pre-stretch of stress fibers generated by actomyosin motors is approximately $0.1,{ }^{41}$ which is commonly regarded as a set-point of strain in cell. Secondly, the cyclic stretching load was applied to the matrix which was then transferred to the cytoskeleton through the FAs, which induces shear stress on FAs and defor- mation of stress fibers. The stability of FAs depending on the forces acted on them was then predicted according to Eqs. (8) and (9). The simultaneous equations system of equilibriums of the cell and matrix were solved by using the Newton-Raphson method at each loading step. When the FAs were disassembled, 
the stress fibers performed depolymerization according to Eq. (10), and the depolymerization rate depends on the magnitude of the external force. On the other side, if the FAs were stable, the stress fiber associated with the stable FAs performed polymerization. The evolution of the system was simulated by simultaneously calculating the deformation of cell-matrix system and the dynamics of turnover of FAs and stress fibers. In each simulation, $N_{\mathrm{t}}=3000$ steps of loading were simulated. To examine the effect of loading frequency on the cell reorientation behaviors, the matrix was loaded at various frequencies.

We used the order parameter of the stress fibers in cell to characterize the cell orientation suggested by previous studies, ${ }^{31}$ which is defined as

$$
S=\langle\cos 2 \theta\rangle=\frac{1}{L_{\text {total }}} \sum_{i=1}^{N_{\mathrm{s}}} l_{\mathrm{s}}(i) \cos 2 \theta_{i}
$$

where $\theta_{i}$ is the direction of the $i$ th stress fiber, $L_{\text {total }}$ is total length of stress fibers in the cell, therefore $l_{\mathrm{s}}(i) / L_{\text {total }}$ is the density function of the stress fiber.

\section{FEM Model}

In our finite element model, we discretized the matrix with rod elements in a triangular lattice (see Fig. 1c). The connecting points of the rods are nodes. There are about 6000 nodes and 18000 rod elements in the matrix. The stress fiber was modeled by the string element, which can only sustain the tension. For each stress fiber, one end was connected with the mass center of cell, and the other end was connected with a lattice node (i.e., the rod end) at the peripheral area of cell. There are 72 stress fibers in the cell model. A user generated code written using FORTRAN program was used for discretization of system.

\section{RESULTS}

\section{Frequency Dependent Disassembly of a Single FA}

The time for disassembling a single FA is one of the key factors that determine the cell reorientation time. Figure 4 shows the dependence of the collapsing time of single FA on the loading frequency. There is a power law relationship between the collapsing time and the loading frequency, as observed in experiments. ${ }^{31}$ The larger the frequency, the faster did the FA collapse. However, if the frequency was lower enough, the collapsing time became infinite large and the FA could keep its stability. ${ }^{34,36}$ There also exists a threshold value of the frequency $(\sim 1 \mathrm{~Hz})$, beyond which the focal adhesion collapsed quickly and the collapsing time remained nearly at a constant value,

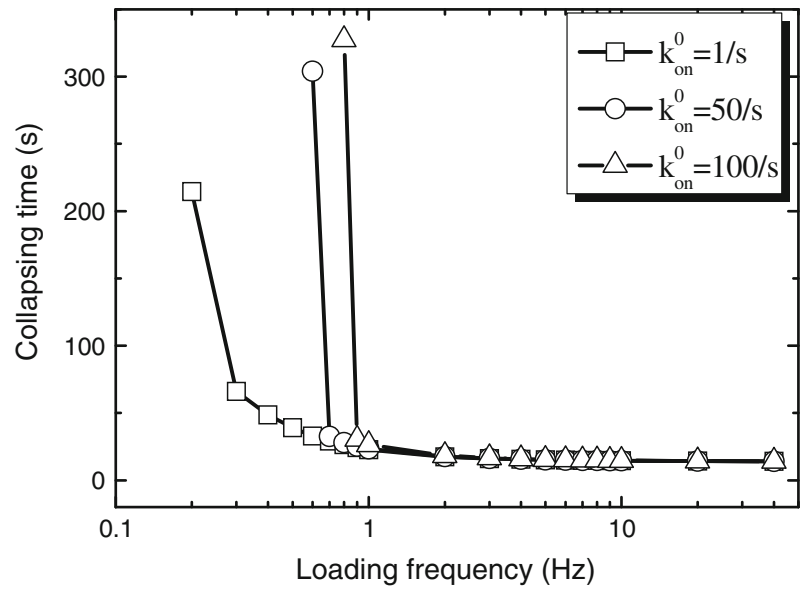

FIGURE 4. The relationship between the collapsing time of FA and the loading frequency predicted by the microscopic bond cluster model. In the calculations, we chose $k_{\text {off }}^{0}=$ $1 \mathrm{~s}^{-1}, \lambda=0.05 \mathrm{~nm}, \varepsilon_{0}=0.05, k_{\mathrm{b}}=10 \mathrm{nN} / \mu \mathrm{m}, k_{\mathrm{s}}=45 \mathrm{nN} / \mu \mathrm{m}$, and $\mu=45 \mathrm{nN} \mathrm{s} \mu \mathrm{m}^{-1}$.

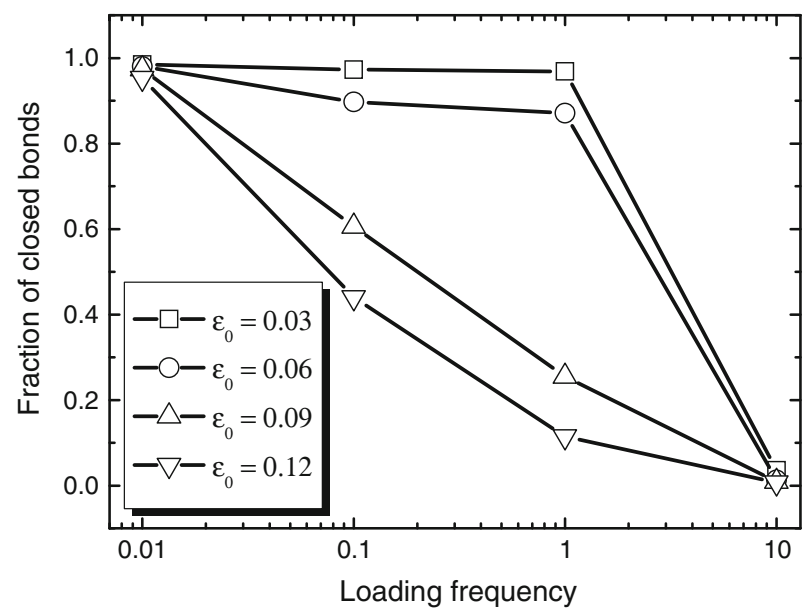

FIGURE 5. The dependence of the mean fraction of closed bonds on the strain amplitude $\varepsilon_{0}$ at different loading frequencies. In the calculation, we chose $k_{\mathrm{on}}^{0}=100 \mathrm{~s}$, and $\varepsilon_{0}=0.03,0.06,0.09$ and 0.12 . The other parameters adopted the same values as those in Fig. 4.

not depending on the frequency any more. After the FA's rupture, the associated stress fibers lost their anchors to matrix and performed depolymerization.

The dependence of mean fraction of closed bonds on the frequency as a function of strain amplitude is depicted in Fig. 5. As can be seen, for small strain amplitude, there is a transition occurring at about $1 \mathrm{~Hz}$ above which the mean fraction of bonds dropped abruptly and then the FA disassembled. However, for high strain amplitude, there is an approximately linear relationship between the mean fraction of bonds and the loading frequency. Therefore, the collapsing time is a function of not only the loading frequency but also the strain amplitude. When the strain amplitude is larger than a threshold value, the collapsing time 
decreased as the strain amplitude increases. The threshold value of loading strain is on the order of a few percent. These results are consistent with the experimental results, ${ }^{16,48}$ suggesting that the collapse of focal adhesion might be closely related with the cell reorientation.

\section{Frequency Dependent Cell Reorientation}

The dynamic process of cell reorientation was simulated using the FEM-based mechano-chemical coupling simulations. Figure 6 shows the responses of stress fibers of the cell to the cyclic stretching at different frequencies. We can see that at low loading frequency, e.g., $\omega=0.1 \mathrm{~Hz}$, the cell was stable without reorientation. In addition, the stretching helped the spreading of the cell with growth of the stress fibers. However, as the frequency was increased up to $\omega=1 \mathrm{~Hz}$, cell reorientation happened, and a lot of stress fibers became perpendicular to the loading direction. As the frequency was further increased to, e.g., $\omega=5 \mathrm{~Hz}$, more stress fibers became perpendicular to the loading direction.

Figure 7 shows the evolution of the orientation of stress fibers (order parameter $S$ ) during the cyclic stretching. As we can see, the rate of cell reorientation and the final alignment of cells are frequency dependent. When the loading frequency was smaller than a lower limiting value, i.e., $\omega=0.1 \mathrm{~Hz}$, the order parameter of cell did not change with time, and the order parameter curve was a horizontal line. When the frequency was increased to be larger than $0.1 \mathrm{~Hz}$, the cell started to reorient and the order parameter decreased. The higher the loading frequency, the faster the rate of reorientation. However, when the loading frequency reached an upper limiting value, i.e., $1 \mathrm{~Hz}$, the curves of order parameter became convergent.

We further calculated the depolymerization rate of stress fibers of different aligning directions (represented by the angles between the stress fibers and the loading direction) at different loading frequencies as shown in Fig. 8. We can see that the smaller the aligning angle, the higher the depolymerization rate. The stress fibers aligning along the loading direction had the maximum depolymerization rate, while the stress fibers perpendicular to the loading direction had the minimum depolymerization rate. There is also a threshold value of the loading frequency for each curve beyond which the stress fiber started to depolymerize. This threshold value increases with the increase of aligning angle of stress fiber.

Figure 9 shows the reorientation time of cell (the time needed for cell to complete the reorientation) vs. the loading frequency. We can see that the behaviors of cell reorientation exhibited a biphasic manner with respect to the loading frequency. Although the reorientation time decreased as the loading frequency increased in an exponential function at the low loading frequency regime, the loading frequency did not influence the reorientation time when it was increased to be higher than an upper limiting value around $1 \mathrm{~Hz}$.

Moreover, the effect of the viscosity coefficient of stress fiber on the reorientation time of cells was calculated. Here the changing of viscosity coefficient was aimed to consider the effect of structural change of cytoskeleton induced by the changing of applied loading, or adhesion state or cell types. We found that the orientation rate increased with the increase of the viscosity coefficient (see Fig. 10). Moreover, the effect of stiffness of matrix on the reorientation of cells was studied, as shown in Fig. 11. As we can see, the cells preferred to reorientate on soft matrix, and the lower the matrix's stiffness, the higher the reorientation rate.

\section{DISCUSSIONS}

To understand the behaviors of cell reorientation under different loading frequencies, the mechanical models considering mechano-chemical coupling were developed at two different length scales, i.e., one is the subcellular FA level, and the other one is the cellular level. At the FA level, the integrin-ligand bonds were modeled as a bond cluster. And at the cellular level, the cell-matrix system was modeled with a mechanochemical coupling continuum model. These two models handshake at the interface between FAs and their associated stress fibers. The mechanical responses of cell and matrix as well as the cell-matrix interaction were simulated by using a FEM-based mechanochemical coupling simulation method, which considered the stability of FAs and remodeling of stress fibers. The reorientation of cell was represented by the collective reorientation and redistribution of the stress fibers in the cell.

\section{Molecular Level Behaviors}

Our stochastic simulation of the bond cluster stability showed that there is a critical value of loading frequency, below which the cluster was stable. However, once the loading frequency was higher than the threshold value, the bond cluster would lose its stability. The higher the loading frequency, the fast did the FA lose its stability as shown in Figs. 4 and 5.

There are two mechanisms for the frequency dependent stability of FAs. One is that the loading rate determines the contact time between the free end of ruptured bonds and the matrix, which in turn influences the forward rate according to Eq. (2). The rapid deformation of the substrate was not favored by the 

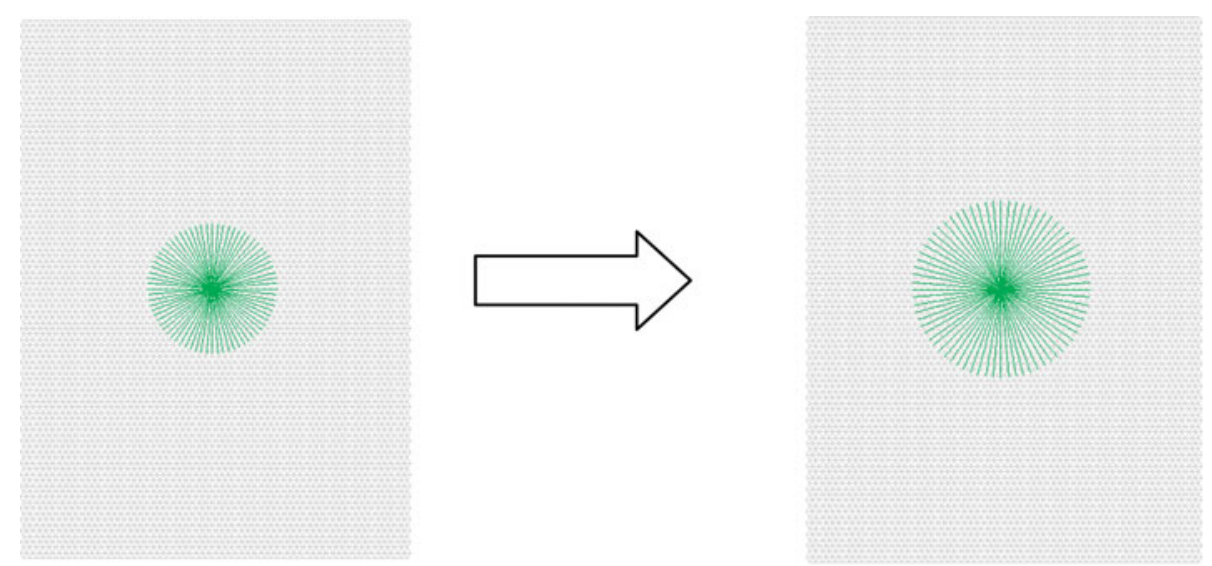

\section{$0.1 \mathrm{~Hz}$}
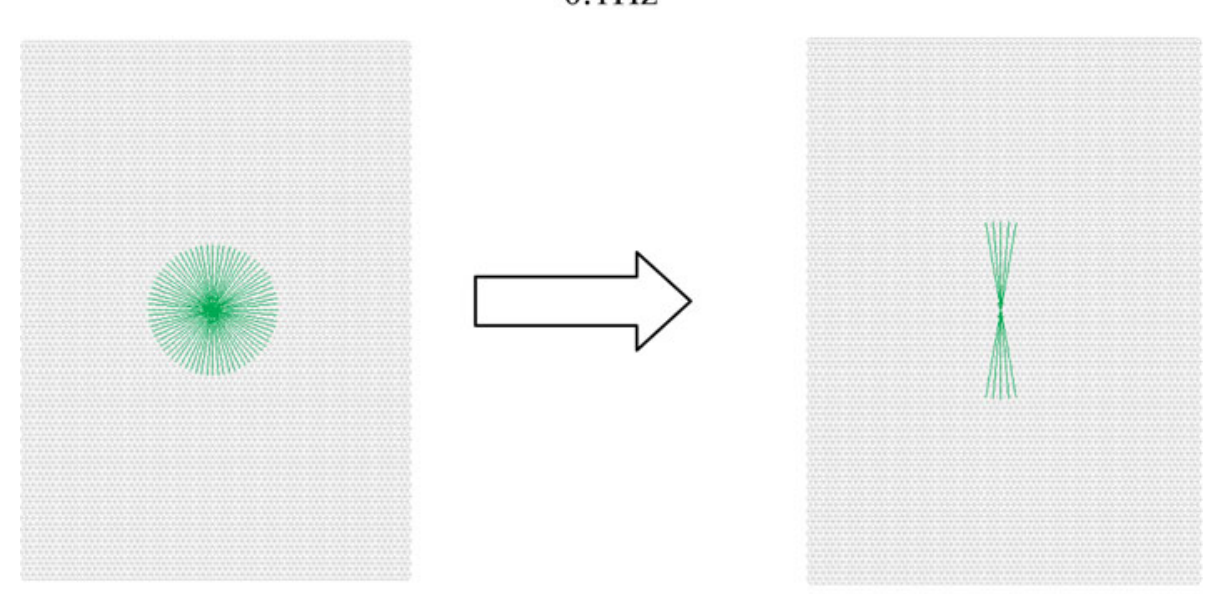

$1.0 \mathrm{~Hz}$
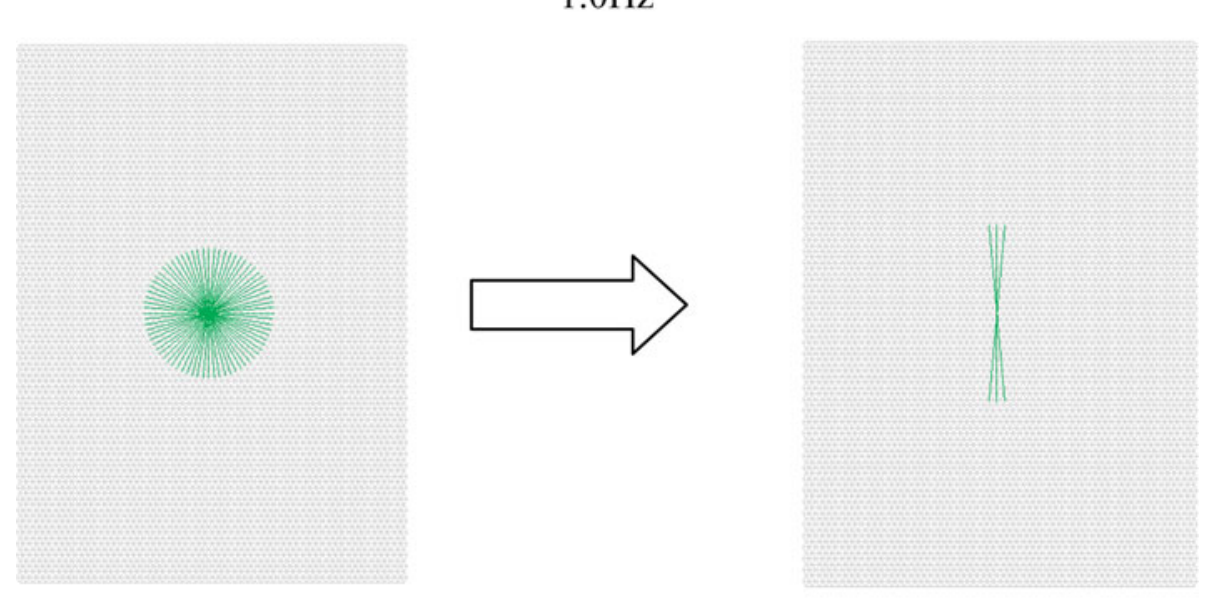

$5.0 \mathrm{~Hz}$

FIGURE 6. The evolution of alignment and distribution of stress fibers in the cell in response to the cyclic stretching at different loading frequencies.

rebinding of a ruptured bond, because the adhesion molecules did not have enough time to contact. The other one is that the deformation of stress fiber was rate dependent due to its intrinsic viscosity. At the low loading frequency, the deformation in stress fibers induced by the substrate stretching was relaxed via its viscoelastic relaxation, i.e., the stress fibers became so soft that they performed large deformation, while the integrin-ligand bonds performed small deformation (because the combination of deformation of the stress fiber and the bonds accommodates the matrix deformation induced by the cyclic stretching), therefore the 


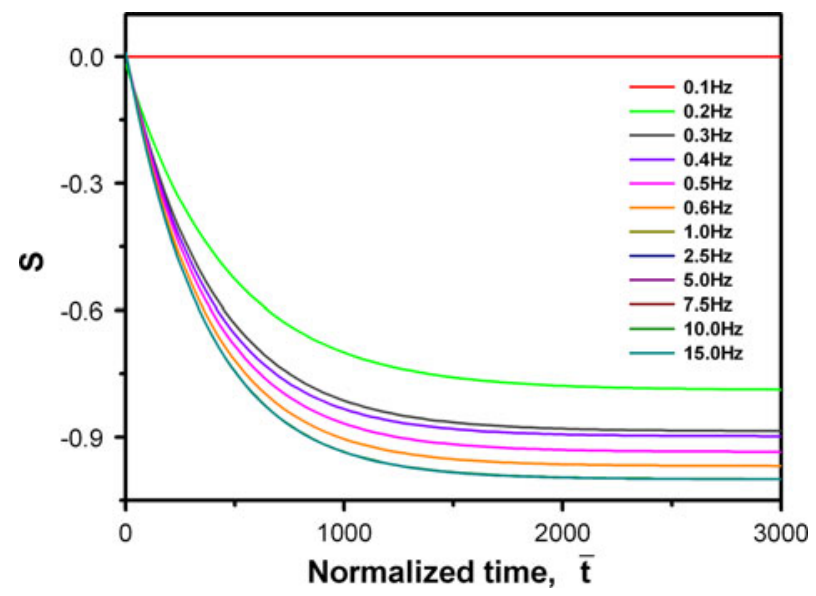

FIGURE 7. The evolution of the order paramter $S$ of actin cytoskeleton (stress fibers) under the cyclic stretching at different loading frequencies. $\bar{t}=t / \Delta t$.

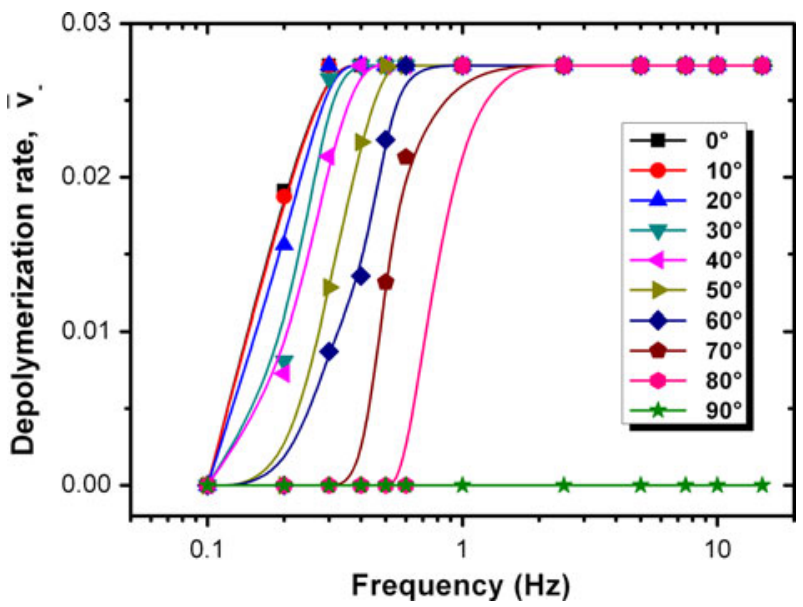

FIGURE 8. The effect of the loading frequency on the depolymerization rate of stress fibers of different aligning directions.

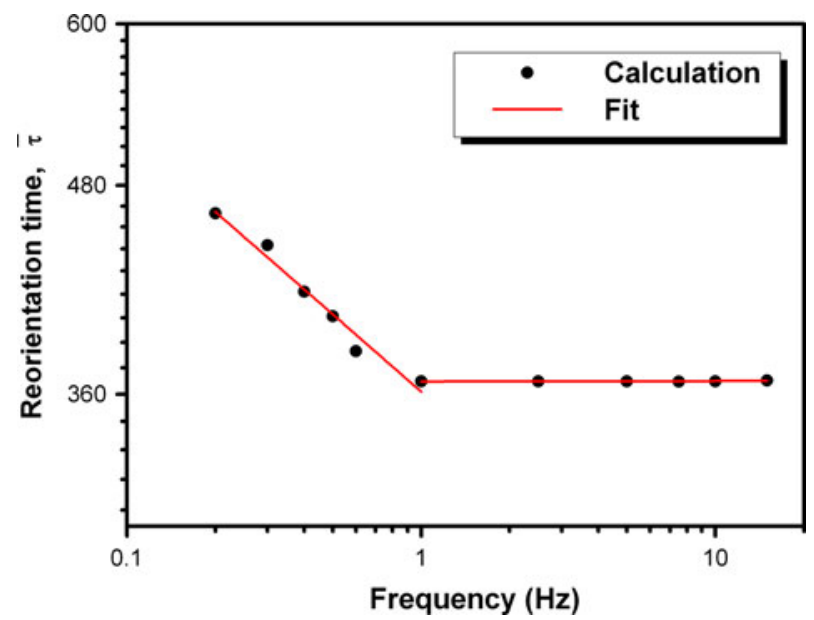

FIGURE 9. The effect of the loading frequency on the reorientation time of cell. The response of the cell reorientation exhibits a biphasic manner to the loading frequency. $\bar{\tau}=\tau / \Delta t$.

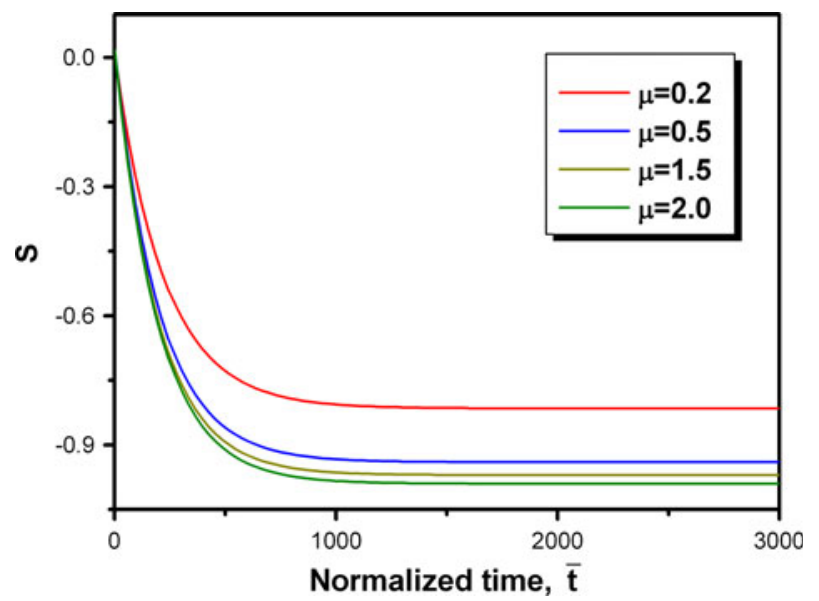

FIGURE 10. Illustration of the effect of the viscosity coefficient of stress fibers on the reorientation behavior of the cell.

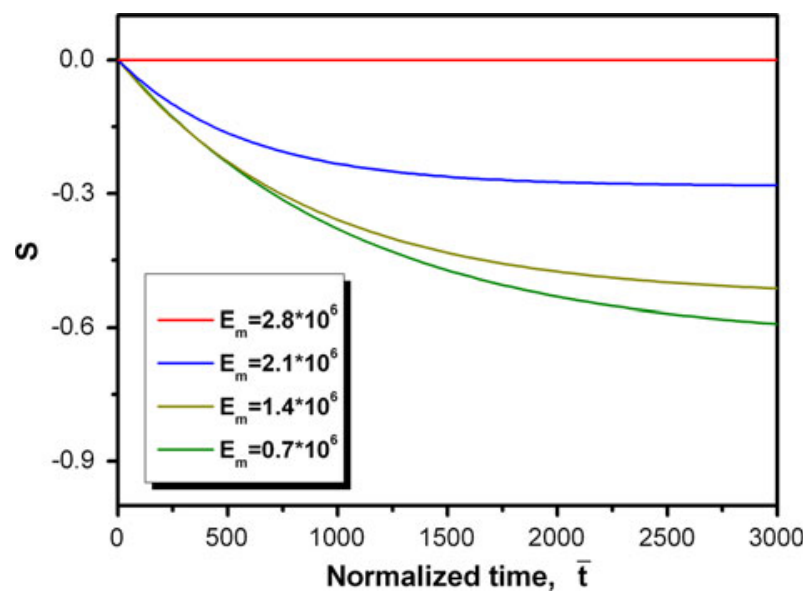

FIGURE 11. The effect of the matrix stiffness on the reorientation of the cell, which shows that the cell prefers to reorientate on the soft matrix.

bonds were stable. In contrast, at high loading frequency, the stress fibers became stiffer than at low loading frequency due to their viscoelastic stiffening. Because of the increase in stiffness of the stress fibers, the integrin-ligand bonds would undergo larger deformation, and became more susceptible to the external load. We found that there is also a threshold of loading strain for disassembly of the cluster, which is on the order of a few percent in consistence with the that of cell reorientation in experiments, ${ }^{16,48}$ suggesting that the collapse of focal adhesion might be related to the reorientation of cells under cyclic stretching.

The mechanics of stability of the FA can also be understood by looking at the behaviors of a single bond. When the external strain was small, the forward rate (bond formation) was much larger than the reverse rate (bond rupture), therefore the bond was stable. However, with increasing of the applied strain, 
the reverse rate was increased because of the increase of bond force according to Eq. (1). There exists a critical value of bond force at which the reverse rate was equal to the forward rate. When the bond force became larger than the critical value, the bond rupture dominated and the bond made a spontaneous state transition from a stable to an unstable state. The physical mechanism is that the action of external force lowered the energy barrier for the bond rupture and made it easier to escape out of the energy well. Therefore, when the strain amplitude of stretching was larger than the threshold, more and more bonds lost stability and finally caused the collapse of the focal adhesion.

\section{Cell Level Behaviors}

At the cell level, cell reorientation was the results of the interplay among the stability of focal adhesion, the turnover of stress fiber, and the cell-matrix interaction.

The cells remained stable instead of reorientation when the loading frequency was under a lower limit. The reason is that the FAs were stable at such low loading frequency, ${ }^{30,36,38}$ and so were the stress fibers associated with the FAs. According to our mechanical model, the force applied on the cell by the cyclic stretching is rate dependent because of the viscoelasticity of cytoskeleton. When the stretching frequency is smaller than or equal to $0.1 \mathrm{~Hz}$, the force applied on the cell is too small to cause the cell reorientation, therefore the curve of $S$ is a constant line. Experiments showed that this lower limit of frequency of cell reorientation was in the range of $0.01-0.1 \mathrm{~Hz}$. For instance, it is about $0.01 \mathrm{~Hz}$ for rat embryonic fibroblasts and about $0.1 \mathrm{~Hz}$ for human dermal fibroblasts, ${ }^{31}$ and also $0.01 \mathrm{~Hz}$ for Bovine aortic endothelial cells. ${ }^{28}$ It seems that different kinds of cells have different sensibilities to the loading frequency.

In contrast, once the loading frequency was increased to be higher than the lower limit, the FAs started to lose their stability at the increased loading frequency, as shown in Fig. 4. Once the FAs ruptured, the tension force in the associated stress fibers would drop abruptly, and then caused their depolymerization and triggered the polymerization of stress fibers in other directions, which made the reorientation of cells. We found that at a comparably low frequency region, the cell reorientation time decreased as the increase of the loading frequency. The mechanism might be that the collapsing time of FAs is much larger than the polymerization time of stress fibers, therefore the collapsing time of FAs dominates the cell reorientation time.

However, further increasing the loading frequency when it was larger than an upper limit around $1 \mathrm{~Hz}$, the cell exhibited a different behavior. The increase of the loading frequency would not further reduce the cell reorientation time, instead the time-frequency curve reached a plateau and the reorientation time became frequency independent, as shown in Fig. 9. The mechanism might be that at this high loading frequency region, the disassembly process of FAs was so fast that their collapsing time became much smaller than the time needed for depolymerization of stress fibers associated with the collapsed FAs as well as that for polymerization of stress fibers associated with the newly formed FAs. Therefore, the cell reorientation exhibited a biphasic character. This phenomenon might be the results of the competition between the dynamics of stability of FAs and the depolymerization and polymerization of stress fibers. At low loading frequency region, the stability of FA dominants the reorientation process, but at the high loading frequency region, the dynamics of stress fiber dominates the reorientation process. This results are consistent with the experiments by Jungbauer et al. ${ }^{31}$

It is noteworthy that this study for the first time simulated the cell reorientation using a multiscale modeling approach that considered the stability of focal adhesion at molecular level, the turnover of the stress fiber at subcellular level, and then cell reorientation at the cellular level. The connection between the molecular to the subcellular levels was made through a probability function of disassembly of FAs which was derived through a stochastic simulation of stability of FAs modeled by the integrin-ligand bond cluster. The subcellular level was connected with the cellular level through the association of stress fiber with the FAs. The collective behaviors of dynamics of the stress fibers produced the cell reorientation as a whole picture. In addition, compared with previous studies, this study considered the elasticity of matrix and viscoelasticity of stress fiber, therefore more precisely considered the cell-matrix interaction. We showed that the matrix stiffness affects the stability of cell adhesion and cell reorientation. For example, cells on softer matrix were less stable and more preferred to reorient under the cyclic stretching. This result is consistent with the experiments that cell could not form stable adhesion on the soft matrix, and the size of FA was smaller than that on stiff matrix. ${ }^{22}$

We would also like to point out that in the present study we only modeled the behavior of single cell without considering the cell-cell interaction, which corresponds to the behaviors of subconfluent cells in the experiments. However, it was found that the cell reorientation behavior does not exhibit the biphasic phenomenon for the confluent cells in the experiments. Their reorientation time always decreases with the increase of the loading frequency. The reason for this 
observation might be that the cell-cell interaction, through the mechanical and chemical signals, would help accelerate the dynamics of the turnover of actin cytoskeleton, including the polymerization and depolymerization of stress fibers, which largely postpone the reaching of the plateau of the curve of reorientation time vs. loading frequency.

\section{CONCLUSIONS}

The frequency dependent stability and reorientation of adherent cells were studied using a multiscale simulation framework. This framework can consider the dynamics of cells from the molecular level to the cell level with a vision that the cell behaviors can be understood from the molecular scale up. The connection between the molecule level and cellular level was made through a subcellular modeling of FAs via a bond cluster model. At each level of the framework, the mechano-chemical coupling was considered, e.g., the effect of force on bond rupture and formation, the effect of force on stability of FAs, and the effect of force on polymerization and depolymerization of stress fibers. This multiscale modeling effort provides many insights into the mechanisms of responses of adherent cells to the external load. We showed that the cell reorientation is an active response of cells to alleviate the excess tension generated by the cyclic stretching in order to maintain an optimum state of cytoskeleton tension.

We found that there are two crucial factors in the cell reorientation behaviors, one is the stability of FAs, and the other one is the viscoelastic properties and the dynamics of polymerization and depolymerization of stress fibers. These two factors both can be affected by the frequency of the cyclic stretching. We showed that the higher the loading frequency, the faster did the FAs lose their stability, because stress fibers became stiffer at high loading frequency which caused larger bond force in the integrin-ligand bonds. Moreover, the rebinding of integrin-ligand bond became more difficult. Once the FAs lost their stability and disassembled, the associated stress fibers would perform depolymerization, while stress fibers in other directions would perform polymerization. Two characteristic time scales will determine the cell reorientation time, one is the collapsing time of FAs, and the other is the polymerization time of stress fibers associated with the newly formed FAs. The collapsing time of FA decreases with the increase of the loading frequency, however the polymerization of stress fibers in the direction with stable FAs will be less affected by the loading frequency. The competition between the collapsing time and the polymerization time at different loading frequency might be the mechanism causing the biphasic behavior of the cell reorientation.

\section{ACKNOWLEDGMENTS}

This research was supported by the National Natural Science Foundation of China through Grant No. 10732050, 10872115 and 11025208. LD acknowledges the support from the key project of Chinese Academy of Sciences through KJCX2-YW-M04 and KJCX-SW-L08.

\section{CONFLICT OF INTEREST}

The authors state that they have no any financial, professional and personal conflict of interests.

\section{REFERENCES}

${ }^{1}$ Arnold, M., E. A. Cavalcanti-Adam, R. Glass, J. Blummel, W. Eck, M. Kantlehner, H. Kessler, and J. P. Spatz. Activation of integrin function by nanopatterned adhesive interfaces. Chemphyschem 5:383-388, 2004.

${ }^{2}$ Aroush, D. R. B., R. Zaidel-Bar, A. D. Bershadsky, and H. D. Wagner. Temporal evolution of cell focal adhesions: experimental observations and shear stress profiles. Soft Matter 4:2410-2417, 2008.

${ }^{3}$ Balaban, N. Q., U. S. Schwarz, D. Riveline, P. Goichberg, G. Tzur, I. Sabanay, D. Mahalu, S. A. Safran, A. Bershadsky, L. Addadi, and B. Geiger. Force and focal adhesion assembly: a close relationship studied using elastic micropatterned substrates. Nat. Cell Biol. 3:466472, 2001.

${ }^{4}$ Bell, G. I. Models for the specific adhesion of cells to cells. Science 200:618-627, 1978.

${ }^{5}$ Bell, G. I., M. Dembo, and P. Bongrand. Cell adhesion. Competition between nonspecific repulsion and specific bonding. Biophys. J. 45:1051-1064, 1984.

${ }^{6}$ Beningo, K. A., M. Dembo, I. Kaverina, J. V. Small, and Y.-L. Wang. Nascent focal adhesions are responsible for the generation of strong propulsive forces in migrating fibroblasts. J. Cell Biol. 153:881-888, 2001.

${ }^{7}$ Bershadsky, A. D., N. Q. Balaban, and B. Geiger. Adhesion-dependent cell mechanosensitivity. Ann. Rev. Cell Dev. Biol. 19:677-695, 2003.

${ }^{8}$ Bershadsky, A., M. Kozlov, and B. Geiger. Adhesionmediated mechanosensitivity: a time to experiment, and a time to theorize. Curr. Opin. Cell Biol. 18:472-481, 2006.

${ }^{9}$ Boal, D. Mechanics of the Cell. Cambridge, UK: Cambridge University Press, 2002.

${ }^{10}$ Brown, E. J. Adhesive interactions in the immune system. Trends Cell Biol. 7:289-295, 1997.

${ }^{11}$ Cavalcanti-Adam, E. A., T. Volberg, A. Micoulet, H. Kessler, B. Geiger, and J. P. Spatz. Cell spreading and focal adhesion dynamics are regulated by spacing of integrin ligands. Biophys. J. 92:2964-2974, 2007. 
${ }^{12}$ Chen, S., and H. Gao. Non-slipping adhesive contact of an elastic cylinder on stretched substrates. Proc. R. Soc. A 462:211-228, 2006.

${ }^{13}$ Chowdhury, F., S. Na, O. Collin, B. Tay, F. Li, T. Tanaka, D. E. Leckband, and N. Wang. Is cell rheology governed by nonequilibrium-to-equilibrium transition of noncovalent bonds? Biophys. J. 95:5719-5727, 2008.

${ }^{14}$ Collinsworth, A. M., C. E. Torgan, S. N. Nagda, R. J. Rajalingam, W. E. Kraus, and G. A. Truskey. Orientation and length of mammalian skeletal myocytes in response to a unidirectional stretch. Cell Tissue Res. 302:243-251, 2000.

${ }^{15}$ Costa, K. D., W. J. Hucker, and F. C. P. Yin. Buckling of actin stress fibers: a new wrinkle in the cytoskeletal tapestry. Cell Motil. Cytoskel. 52:266-274, 2002.

${ }^{16}$ Dartsch, P., and H. Hammerle. Orientation response of arterial smooth muscle cells to mechanical stimulation. Eur. J. Cell Biol. 41:339-346, 1986.

${ }^{17}$ De, R., A. Zemel, and S. A. Safran. Dynamics of cell orientation. Nat. Phys. 3:655-659, 2007.

${ }^{18}$ Deguchi, S., T. Ohashi, and M. Sato. Tensile properties of single stress fibers isolated from cultured vascular smooth muscle cells. J. Biomech. 39:2603-2610, 2006.

${ }^{19}$ Eastwood, M., V. C. Mudera, D. A. McGrouther, and R. A. Brown. Effect of precise mechanical loading on fibroblast populated collagen lattices: morphological changes. Cell Motil. Cytoskel. 40:13-21, 1998.

${ }^{20}$ Erdmann, T., and U. S. Schwarz. Stability of adhesion clusters under constant force. Phys. Rev. Lett. 92:108102, 2004.

${ }^{21}$ Filippov, A. E., J. Klafter, and M. Urbakh. Friction through dynamical formation and rupture of molecular bonds. Phys. Rev. Lett. 92:135503, 2004.

${ }^{22}$ Geiger, B., J. P. Spatz, and A. D. Bershadsky. Environmental sensing through focal adhesions. Nat. Rev. Mol. Cell Biol. 10:21-33, 2009.

${ }^{23}$ Gerthoffer, W. T., and S. J. Gunst. Signal transduction in smooth muscle-invited review: focal adhesion and small heat shock proteins in the regulation of actin remodeling and contractility in smooth muscle. J. Appl. Phys. 91:963972, 2001.

${ }^{24}$ Goffin, J. M., P. Pittet, G. Csucs, J. W. Lussi, J.-J. Meister, and B. Hinz. Focal adhesion size controls tension-dependent recruitment of $\{$ alpha $\}$-smooth muscle actin to stress fibers. J. Cell Biol. 172:259-268, 2006.

${ }^{25}$ Gumbiner, B. M. Cell adhesion: the molecular basis of tissue architecture and morphogenesis. Cell 84:345-357, 1996.

${ }^{26}$ Hansen, J. C., R. Skalak, S. Chien, and A. Hoger. An elastic network model based on the structure of the red blood cell membrane skeleton. Biophys. J. 70:146-166, 1996.

${ }^{27}$ Hotulainen, P., and P. Lappalainen. Stress fibers are generated by two distinct actin assembly mechanisms in motile cells. J. Cell Biol. 173:383-394, 2006.

${ }^{28}$ Hsu, H. J., C. F. Lee, and R. Kaunas. A dynamic stochastic model of frequency-dependent stress fiber alignment induced by cyclic stretch. PLOS ONE 4(3):e4853, 2009.

${ }^{29} \mathrm{Ji}$, B., and G. Bao. Cell and molecular biomechanics: perspectives and challenges. Acta Mechanica Solida Sinica 24:27-51, 2011 .

${ }^{30} \mathrm{Ji}$, B., D. Kong, and L. H. Dai. Dynamics of adhesion cluster and cell reorientation under lateral cyclic loading. Biorheology 45:96-97, 2008.

${ }^{31}$ Jungbauer, S., H. Gao, J. P. Spatz, and R. Kemkemer. Two characteristic regimes in frequency-dependent dynamic reorientation of fibroblasts on cyclically stretched substrates. Biophys. J. 95:3470-3478, 2008.
${ }^{32}$ Kaunas, R., and H.-J. Hsu. A kinematic model of stretchinduced stress fiber turnover and reorientation. J. Theor. Biol. 257:320-330, 2009.

${ }^{33}$ Kaunas, R., Z. Y. Huang, and J. Hahn. A kinematic model coupling stress fiber dynamics with JNK activation in response to matrix stretching. J. Theor. Biol. 264:593-603, 2010.

${ }^{34}$ Kaunas, R., P. Nguyen, S. Usami, and S. Chien. From the cover: cooperative effects of Rho and mechanical stretch on stress fiber organization. Proc. Natl Acad. Sci. USA. 102:15895-15900, 2005.

${ }^{35}$ Kong, F., A. J. Garcia, A. P. Mould, M. J. Humphries, and C. Zhu. Demonstration of catch bonds between an integrin and its ligand. J. Cell Biol. 185:1275-1284, 2009.

${ }^{36}$ Kong, D., B. Ji, and L. Dai. Stability of adhesion clusters and cell reorientation under lateral cyclic tension. Biophys. J. 95:4034-4044, 2008.

${ }^{37}$ Kong, D., B. Ji, and L. Dai. Nonlinear mechanical modeling of cell adhesion. J. Theor. Biol. 250:75-84, 2008.

${ }^{38}$ Kong, D., B. H. Ji, and L. H. Dai. Stabilizing to disruptive transition of focal adhesion response to mechanical forces. J. Biomech. 43:2524-2529, 2010.

${ }^{39}$ Krasik, E. F., and D. A. Hammer. A semianalytic model of leukocyte rolling. Biophys. J. 87:2919-2930, 2004.

${ }^{40}$ Kumar, S., I. Z. Maxwell, A. Heisterkamp, T. R. Polte, T. P. Lele, M. Salanga, E. Mazur, and D. E. Ingber. Viscoelastic retraction of single living stress fibers and its impact on cell shape, cytoskeletal organization, and extracellular matrix mechanics. Biophys. J. 90:3762-3773, 2006.

${ }^{41}$ Lan, L., F. Yunfeng, J. H. William, J. O. Sara, D. L. Gregory, and C. P. Y. Frank. Actin stress fiber pre-extension in human aortic endothelial cells. Cell Motil. Cytoskel. 65:281-294, 2008.

${ }^{42}$ Lawrence, M. B., and T. A. Springer. Leukocytes roll on a selectin at physiologic flow rates: distinction from and prerequisite for adhesion through integrins. Cell 65:859873, 1991.

${ }^{43}$ Lee, C. F., C. Haase, S. Deguchi, and R. Kaunas. Cyclic stretch-induced stress fiber dynamics-dependence on strain rate, Rho-kinase and MLCK. Biochem. Biophys. Res. Commun. 401:344-349, 2010.

${ }^{44}$ Liu, B., M.-J. Qu, K.-R. Qin, H. Li, Z.-K. Li, B.-R. Shen, and Z.-L. Jiang. Role of cyclic strain frequency in regulating the alignment of vascular smooth muscle cells in vitro. Biophys. J. 94:1497-1507, 2008.

${ }^{45}$ Maheshwari, G., G. Brown, D. A. Lauffenburger, A. Wells, and L. G. Griffith. Cell adhesion and motility depend on nanoscale RGD clustering. J. Cell. Sci. 113:1677-1686, 2000.

${ }^{46}$ Marshall, B. T., M. Long, J. W. Piper, T. Yago, R. P. McEver, and C. Zhu. Direct observation of catch bonds involving cell-adhesion molecules. Nature 423:190-193, 2003.

${ }^{47}$ Matsui, T. S., K. Ito, R. Kaunas, M. Sato, and S. Deguchi. Actin stress fibers are at a tipping point between conventional shortening and rapid disassembly at physiological levels of MgATP. Biochem. Biophys. Res. Commun. 395: 301-306, 2010.

${ }^{48}$ Neidlinger-Wilke, C., H. J. Wilke, and L. Claes. Cyclic stretching of human osteoblasts affects proliferation and metabolism - a new experimental-method and its application. J. Orthopaed. Res. 12:70-78, 1994.

${ }^{49}$ Paul, R., P. Heil, J. P. Spatz, and U. S. Schwarz. Propagation of mechanical stress through the actin cytoskeleton toward focal adhesions: model and experiment. Biophys. $J$. 94:1470-1482, 2008. 
${ }^{50}$ Peterson, L. J., Z. Rajfur, A. S. Maddox, C. D. Freel, Y. Chen, M. Edlund, C. Otey, and K. Burridge. Simultaneous stretching and contraction of stress fibers in vivo. Mol. Biol. Cell 15:3497-3508, 2004.

${ }^{51}$ Rinko, L. J., M. B. Lawrence, and W. H. Guilford. The molecular mechanics of P- and L-selectin lectin domains binding to PSGL-1. Biophys. J. 86:544-554, 2004.

${ }^{52}$ Riveline, D., E. Zamir, N. Q. Balaban, U. S. Schwarz, T. Ishizaki, S. Narumiya, Z. Kam, B. Geiger, and A. D. Bershadsky. Focal contacts as mechanosensors: externally applied local mechanical force induces growth of focal contacts by an mial-dependent and ROCK-independent mechanism. J. Cell Biol. 153:1175-1186, 2001.

${ }^{53}$ Sastry, S. K., and K. Burridge. Focal adhesions: a nexus for intracellular signaling and cytoskeletal dynamics. Exp. Cell Res. 261:25-36, 2000.
${ }^{54}$ Satcher, R. L., and C. F. Dewey. Theoretical estimates of mechanical properties of the endothelial cell cytoskeleton. Biophys. J. 71:109-118, 1996.

${ }^{55}$ Tan, J. L., J. Tien, D. M. Pirone, D. S. Gray, K. Bhadriraju, and C. S. Chen. From the cover: cells lying on a bed of microneedles: an approach to isolate mechanical force. Proc. Natl Acad. Sci. 100:1484-1489, 2003.

${ }^{56}$ Wang, J. H. C., P. Goldschmidt-Clermont, J. Wille, and F. C. P. Yin. Specificity of endothelial cell reorientation in response to cyclic mechanical stretching. J. Biomech. 34:1563-1572, 2001.

${ }^{57}$ Wang, H. C., W. Ip, R. Boissy, and E. S. Grood. Cell orientation response to cyclically deformed substrates: experimental validation of a cell model. J. Biomech. 28:1543-1552, 1995. 